\title{
La atención a las personas en situación de dependencia como yacimiento de empleo en tiempos de crisis: el caso español
}

\author{
José Antonio CAMACHO BALLESTA \\ Profesor Titular del Departamento de Economía Internacional y de España \\ Facultad de CCEE y Empresariales \\ Secretario del Instituto de Desarrollo Regional (IDR) \\ Universidad de Granada \\ jcamacho@ugr.es \\ Soraya María RUIZ PEÑALVER \\ Instituto de Desarrollo Regional (IDR) \\ Universidad de Granada \\ soraya_rp@ugr.es \\ María de los Ángeles MINGUELA RECOVER \\ Instituto de Desarrollo Regional (IDR) \\ Universidad de Granada \\ mminguela@ugr.es
}

Recibido: 20/06/2013

Aceptado: 20/01/2014

\section{RESUMEN}

En las últimas décadas se ha producido un envejecimiento de la población europea y española como consecuencia del movimiento natural de la población. Esta situación justifica una mayor protección social para las personas en situación de dependencia, entre las que se incluyen aquéllas que presentan discapacidad y a las personas mayores por su vulnerabilidad ante situaciones de dependencia. Por ello, en España se han desarrollado servicios vinculados a la atención de estas personas, ya que precisan de cuidados específicos. Estas actividades no sólo cubren esta demanda social creciente, sino que además, han generado empleo en un contexto de crisis, marcado por la destrucción del empleo. El objetivo de este artículo es demostrar la capacidad de generación de empleo por parte de los servicios relacionados con la atención a las personas en situación de dependencia, en un escenario marcado por una profunda crisis con graves consecuencias en el mercado laboral.

Palabras clave: dependencia, envejecimiento, empleo, mercado de trabajo, servicios sociales.

Care giving to dependent people. A source of jobs in times of crisis. The case of Spain

\footnotetext{
ABSTRACT

Ageing of European and Spanish populations in recent decades reflects natural demographic evolution triggered by even greater longevity of senior citizens, and falling birth and fertility rates. Such evolution
} 
warrants greater social protection for dependent people, including not only people with disabilities but also elderly citizens whose dependency makes them vulnerable. Spain's expanding social safety network has identified several economic activities to provide care for dependent people who for various reasons require specific types of attention. Such activities require a growing supply of services to fulfill likewise expanding social demand to meet related specific needs. But they have also, and contrary to many other activities, created jobs despite the present financial and economic crisis marked by job destruction.

Against this backdrop, our paper shows care giving for dependents can create jobs even under conditions of a deep crisis that is having serious consequences in the labour market.

Keywords: dependents, elderly, employment, labor market, social services and employment sources.

\section{REFERENCIA NORMALIZADA}

Camacho Ballesta, J., Ruiz Peñalver, S., Minguela Recover, M.A. (2013). "La atención a las personas en situación de dependencia como yacimiento de empleo en tiempos de crisis: el caso español”. Cuadernos de Relaciones Laborales. Vol. 33, núm.1, p. 169-188.

SUMARIO: Introducción. 1. Factores sociodemográficos de las personas en situación de dependencia en España. 2. El mercado de trabajo en España. 3. El mercado de trabajo relacionado con el Sistema de Autonomía y Atención a la Dependencia (SAAD). 4. Conclusiones. 5. Referencias bibliográficas.

\section{Introducción}

Desde finales de la década de los 80 del siglo pasado, Europa trabaja en la construcción de un modelo social europeo como herramienta de cohesión social, cuyos principios y valores comunes son: la solidaridad, la justicia social, la igualdad de género, el acceso universal a prestaciones y servicios, además de promulgar la igualdad de oportunidades respecto a personas mayores, jóvenes, personas con discapacidad y personas en riesgo de exclusión social (A.o.E., 2005).

Tradicionalmente se han identificado cuatro regímenes de bienestar atendiendo a su localización geográfica (Andersen, 1990; Ferrera, 1996):

Los países nórdicos (Suecia, Finlandia, Dinamarca y Holanda), caracterizados por un modelo Beveridge con un alto en gasto en protección social, siendo su cobertura y acceso universal.

Otros países como Reino Unido e Irlanda, se caracterizan por un modelo liberal en el que la intervención del Estado es garantizar un bienestar mínimo. El acceso a la prestaciones se realizan bajo una prueba de recursos y atendiendo a una serie de criterios estrictos y estigmatizados (Eikemo,et al. 2008; Bambra, 2007; Andersen, 1990).

El modelo continental o Bismarckiano (Alemania, Francia, Bélgica, Austria y Luxemburgo) caracterizado por los seguros sociales, en los que la protección social prestada por el Estado está condicionada a una cotización previa por parte del trabajador, es decir, es financiada a cargo de las cotizaciones entre el trabajador y el empresario. La atención a las personas en situación de dependencia desde la década de los 90 del siglo pasado se ha considerado como un pilar más dentro del sistema la Seguridad Social. Claros ejemplos, son la promulgación en Alemania en el año 1994 de la Ley de Seguro Social de la Dependencia (Gesetz Zur Sozialen Pflegeversicherung) y en Francia en el año 2000, la creación de la Ayuda Personalizada de Autonomía (Allocation personnalisée d'autonomie). 
El modelo mediterráneo (España, Italia, Grecia y Portugal) se caracteriza porque encuentra la importancia y/o centralidad de la familia en la prestación de bienestar social, en cambio, el Estado tiene un papel subsidiario. Se trata de un modelo en el que los dualismos, la fragmentación y la ineficiencia social de sus sistemas de protección han caracterizado al Estado como benefactor con un bajo gasto público en protección social (Ferrera, 1996; Gal, 2010).

Sin embargo, el afán por una modernización de los sistemas de protección social y la construcción de un modelo social europeo común, se topa con la existencia de importantes asimetrías entre los estados miembros como son: la importancia de la familia, el volumen y calidad de los servicios sociales públicos, aspectos financieros relacionados con la sostenibilidad de los sistemas y cambios institucionales como la descentralización, la privatización...etc. (Rodríguez Cabrero, 2007a).

En el caso concreto de España, el sistema de protección social se caracteriza por ser el mejor representante del welfare mixed. Este combina aspectos del modelo bismarckiano y beveridgeano, contributivo y universalista, respectivamente (Moreno Rodríguez, 2001; Kammer, et al. 2012; Camacho Ballesta, et al, 2008).

En el año 2003, se produjo la Renovación del Pacto de Toledo aprobándose la necesidad de configurar un sistema integrado que abordase las situaciones de dependencia atendiendo a las recomendaciones que desde Europa se hacían. Y en el año 2006, se ratificó la Ley 39/2006, de 14 de diciembre, de Promoción de la Autonomía Personal y Atención a las personas en situación de dependencia (LAPAD). El objetivo se centraba en construir, articular y mantener un sistema universal, equitativo y accesible de protección, que diese una respuesta coordinada, global e integral a esta demanda social creciente. De este modo se ha generado un nuevo derecho de ciudadanía de carácter subjetivo, la atención a la dependencia, y además sirve de complemento al fragmentado sistema público de servicios sociales.

La LAPAD además de servir de complemento al fragmentado sistema público de servicios sociales, debía de tener en cuenta de manera transversal los cambios en las estructuras y dinámicas familiares, el cambio en la aspiraciones de las mujeres más allá del ámbito familiar (Beck- Gernsheim, 2011), es decir, debía de responder al sistema de cuidados informales, en el que la mujer ha tenido y tiene un papel protagonista. Como resultado, la LAPAD anexaba el Sistema para la Autonomía y Atención a la Dependencia (SAAD). Desde 2007 se ha producido un aumento de los recursos de naturaleza mixta, la mejora en la accesibilidad y en la cobertura (Rodríguez Cabrero, 2007b), a través de una cartera renovada de recursos junto a una serie de prestaciones económicas de diversa naturaleza: vinculadas al servicio, de asistencia personal, tanto para los cuidados en el entorno familiar como de apoyo a cuidadores no profesionales.

El macroproyecto del bienestar no sólo pretendía la mejora de la calidad de vida de las personas en situación de dependencia, reconocida como riesgo social, sino también la de sus familias, y dentro de ellas, la de las personas cuidadoras no profesionales, generalmente mujeres. Las tareas de cuidados tradicionalmente enmarcados en la esfera privada de los hogares, formando parte del trabajo no remunerado y por tanto, invisibles (Prieto y Serrano, 2013; Carrasco, 2006). 
Así pues, la promulgación de la LAPAD ha reconocido y dignificado la labor que realizan estas personas a través del reconocimiento de una prestación económica para cuidados en el entorno familiar y apoyo a cuidadores no profesionales (Del Pozo Rubio y Escribano Sotos, 2012; García Gómez, et al., 2011).De hecho, la atención a la dependencia se trata de una actividad intensiva en mano de obra, tanto es así que el Libro blanco de atención las personas en situación de dependencia estimaba una creación de unos 300 mil puestos de trabajo directos. Pero en ningún caso, se preveía que desde el año 2008, España iba estar sumida en una profunda crisis económica con graves consecuencias en términos de destrucción de empleo, entre otros aspectos.

Por tanto, mientras avanzan las etapas de implantación del SAAD, el objetivo del presente trabajo es analizar si los servicios relacionados con la atención a las personas en situación de dependencia en España son un yacimiento de empleo durante el periodo comprendido entre 2008-2012, tiempo de inestabilidad de la economía y de destrucción masiva de empleo.

El análisis se divide en dos grandes apartados, en primer lugar, se realiza una breve contextualización del escenario sociodemográfico de la atención a la situación de dependencia en España, en perspectiva comparada con la Unión Europea de los 27 (UE-27) siempre que la información disponible lo ha permitido.

En segundo lugar, se realiza un análisis del mercado del trabajo en España durante los años 2008-2012, periodo en el que comienza y se desarrolla la actual crisis financiera y económica. Esta parte distingue los siguientes aspectos:

Por un lado, se analiza brevemente cuál es la situación en la que se encuentra el mercado de trabajo español en general haciendo especial hincapié en la población desempleada. Para ello se han utilizado datos procedentes de la Encuesta de Población Activa (EPA) (INE, 2013a). En segundo lugar se estudia la situación laboral de las actividades vinculadas con los SAAD durante 2008-2012.

Para finalizar el estudio del mercado de trabajo en España, se ha analizado el número de afiliados en los servicios relacionados con la atención a las personas en situación de dependencia y su presencia en el mercado de trabajo. En esta sección se ha utilizado la información mensual para el periodo 2008-2012 que ofrece la Tesorería General de la Seguridad Social. Resulta imprescindible indicar que los datos de 2008 presentan una metodología diferente al resto de años, ya que las actividades vinculadas a los SAAD aparecen junto a las actividades sanitarias, por lo que las comparaciones de las actividades vinculadas a los SAAD sólo se pueden realizar para 2009-2012.

\section{Factores sociodemográficos de las personas en situación de dependencia en España}

Las situaciones de dependencia son definidas como 
un estado en el que se encuentran las personas que por razones ligadas a la falta o la pérdida de autonomía física, psíquica o intelectual tienen necesidad de asistencia y/o ayudas importantes a fin de realizar los actos corrientes de la vida diaria y, de modo particular, los referentes al cuidado personal

atendiendo a la Recomendación No (98) 9 del Consejo Europeo (CE, 1998: 51). En otras palabras, se trata de la necesidad de recibir ayuda por parte de una tercera persona para la realización de las actividades cotidianas. Estas actividades de la vida cotidiana se distinguen en dos grandes grupos, por un lado, las actividades básicas de la vida diaria (ABVD), como la movilidad, el cuidado personal, y el desplazamiento dentro hogar. Y por otro lado, las actividades instrumentales de la vida diaria (AIVD) como la realización de las tareas del hogar, el desplazamiento fuera del hogar, la preparación de comidas, el cumplimiento de prescripciones médicas (Abellán García, et al., 2011; Kemper, 1992)

La encuesta sobre Discapacidades, Autonomía personal y situaciones de Dependencia publicada en el año 2008 permite contextualizar la situación de las personas en situación de dependencia ${ }^{1}$ en España, ascendiendo al 6,5 \% del total de la población mayor de 6 años. Las situaciones de dependencia se encuentran a lo largo de la toda la pirámide de población (véase fig. 1). Sin embargo, la prevalencia es superior para la población mayor de 65 años, representando al 62,7\% del total de personas en situación de dependencia. La notoriedad de este grupo de población no sólo es debido al aumento de la esperanza de vida al nacer, la cual, se encuentra en torno a los 81,3 años. Si no también, al número de años que una persona va a vivir en un estado de buena salud, es decir, la esperanza de vida libre de discapacidad (healthy life), 63,6 años para las mujeres y 64,1 años para los hombres ambos por encima de la media de la UE-27 (EUROSTAT,2013a). Ambos indicadores proyectan la mejora de la calidad de vida de la sociedad española desde la década de los 90 del siglo pasado.

${ }^{1}$ Para el cálculo de las personas en situación de dependencia se ha seguido la metodología propuesta por Gregorio Rodríguez Cabrero (véase Rodríguez Cabrero, 2004; Esparza Catalán, 2010). 


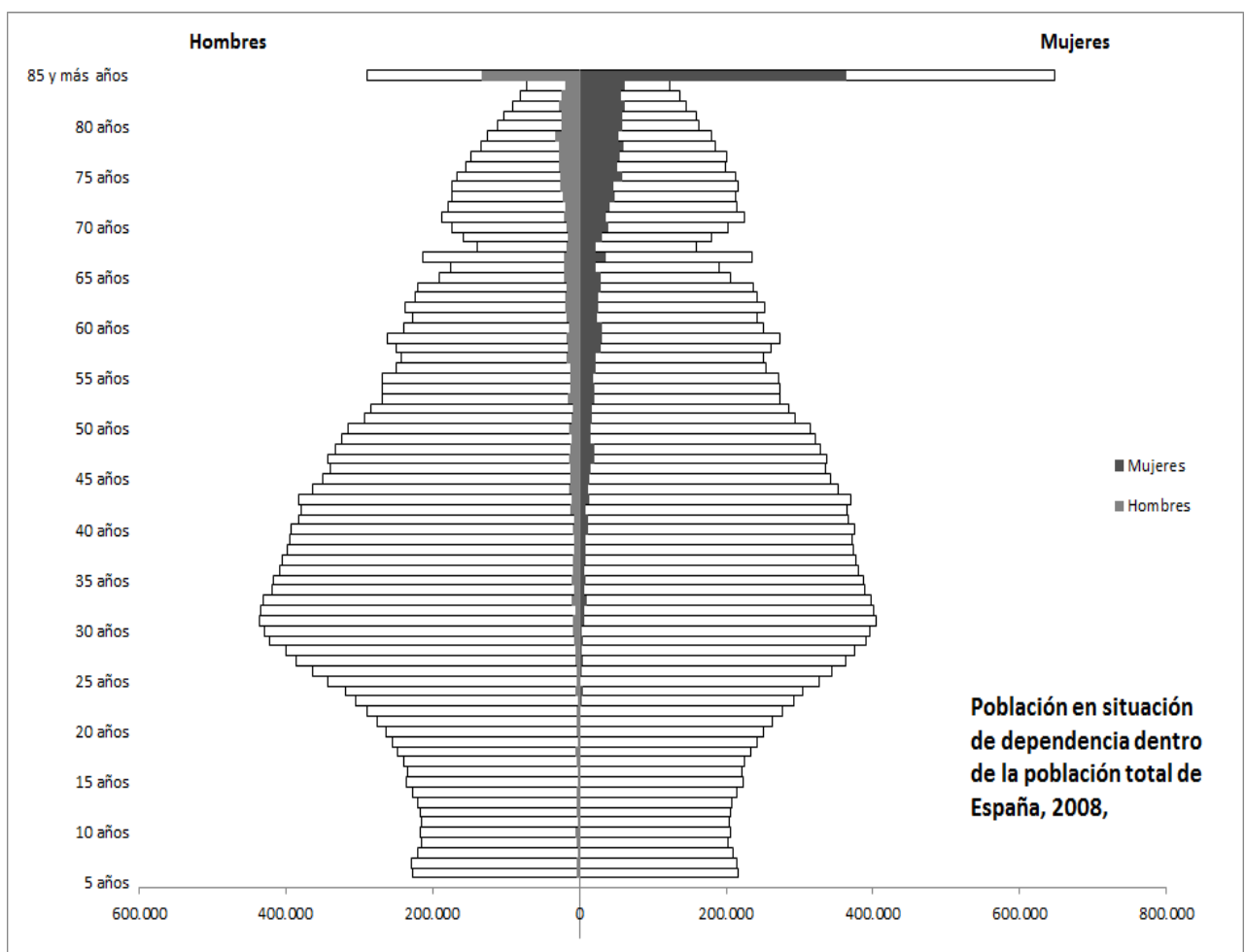

Fig. 1 Población en situación de dependencia dentro del total de la población española en el año 2008.Fuente: Elaboración propia a partir de INE y explotación de los microdatos de la encuesta EDAD, 2008.

Si bien es cierto, no se pueden asociar exclusivamente las situaciones de dependencia a cuestiones de edad, debido a que el 37,3\% de la población menor de 65 años sufre algún tipo de discapacidad o situación de dependencia en el desempeño de las actividades básicas e instrumentales de la vida diaria. Entre la heterogeneidad de las situaciones que originan mermas en la autonomía personal se encuentran: problemas en el parto, enfermedades congénitas, accidentes de tráfico...etc. En este sentido, se van a resaltar aquellas situaciones de dependencia que tiene su origen en las condiciones de trabajo. Los accidentes de trabajo y las enfermedades profesionales son las principales causas que originan situaciones de dependencia y de discapacidad dentro de la población ocupada. Así mismo la franja de edad que abarca desde los 45 a los 64 años agrupa al 44,8\% de personas en situación de dependencia debido a un accidente de trabajo y al $40 \%$ de personas en situaciones de dependencia debido a una enfermedad profesional. Evidentemente, no son las únicas causas que determinan las situaciones de dependencia pero sí son las más comunes.

Finalmente, las previsiones sociodemográficas a largo plazo se dirigen hacia una sociedad tremendamente envejecida, así lo indican las proyecciones oficiales a 2050 
publicadas por Eurostat (Eurostat, 2013b). En una breve comparación con la UE-27, población activa europea (población entre 15-64 años) bajará en 45,2 millones de personas, o sea, un 13,5\%, mientras que la población española para la misma franja de edad, disminuirá en 2,0 millones de personas. Sin embargo, la población de 65 y más años en 2050 se incrementará en un 72,0\% en Europa. En España el aumento será espectacular para 2050 ya que aumentará en 8,9 millones de personas, suponiendo un incremento del $112,0 \%$. Y dentro de este grupo de población el crecimiento más veloz será el de los mayores de 80 años. Todo ello, tendrá una repercusión directa sobre el mercado de trabajo, en dos sentidos, por un lado, se caracterizará por una fuerza de trabajo cada vez más envejecida con el consiguiente aumento de las situaciones de dependencia derivadas de las condiciones de trabajo. Y por otro lado, gran parte del mercado se centrará en la satisfacción de las necesidades derivadas del envejecimiento de la población como de las situaciones de dependencia independientemente de la causa que la originó.

\section{El mercado de trabajo en España}

El mercado de trabajo español se ha visto seriamente perjudicado por la crisis financiera que comenzó en Estados Unidos en 2007, y que se hizo notar en nuestro país aproximadamente en el tercer trimestre de ese mismo año. En España, se ha convertido en una crisis económica debido a las características de nuestro modelo de crecimiento, basado en una economía tradicional bastante dependiente de Europa, algo casi adictivo para algunos sectores como las actividades agroalimentarias, el sector automotor así como las actividades vinculadas con el turismo. Otras actividades económicas como el sector de la construcción que ha sido el gran perjudicado por la crisis financiera, ha tenido gravísimas pérdidas de empleo. Asimismo, esta actividad que está muy vinculada con otras como las industrias minero-extractivas, los fabricantes de materiales de construcción, la metalurgia, los servicios inmobiliarios, los servicios de arquitectura, la fabricación de mobiliario, etc., ha trasladado esa pérdida de empleo de unas a otras actividades.

A su vez cabe destacar que la creciente carga de la deuda de las entidades financieras, seguido por el exceso de endeudamiento de las empresas no financieras, las familias y en última instancia, el Estado, ha sido un importante canal a través del cual la economía española ha sido especialmente contaminada por la crisis financiera internacional. Es de destacar que, aunque menos endeudado que otros agentes económicos en el inicio de la crisis, el Estado se ha convertido en el pagador de último recurso, especialmente debido a los efectos de los estabilizadores automáticos de nuestro Estado de bienestar, en el que los ingresos públicos se han reducido drásticamente, mientras que los costes asociados a la crisis, como las prestaciones por desempleo, las pensiones y otras prestaciones sociales, han crecido considerablemente.

Como ya se ha comentado, la característica esencial a día de hoy del mercado de trabajo español, es la elevada destrucción de empleo provocada en parte por la crisis. 
De hecho, el número de desempleados se considera como un claro indicador de las consecuencias de dicha crisis, el cual ha aumentado en un 174,8\% entre 2008 y 2012. Las cifras oficiales de la EPA (véase INE, 2013a), muestran que el número de parados en España pasó de 2,2 millones de personas en el primer cuatrimestre de 2008, a casi 6,0 millones de parados en el cuarto trimestre de 2012. Sólo se observan ligeras recuperaciones asociadas a los meses estivales, cuando el número de parados se contrae levemente como consecuencia de las contrataciones (normalmente temporales) asociadas con el sector servicios, especialmente vinculadas a las actividades turísticas (restauración, hostelería, etc.).

Por género, se muestra una tendencia creciente y similar para ambos sexos, aunque en términos absolutos el número de hombres parados durante 2008-2012 era mayor que el de mujeres por su mayor participación en el mercado de trabajo. Si bien el número de varones parados en el primer trimestre de 2008 era de 1,0 millón frente a 1,2 millones de mujeres desempleadas, en el cuarto trimestre de 2012 la cifra ascendió a 3,2 millones de hombres frente a 2,8 millones de mujeres, presentando el número de personas desempleadas durante este periodo unas tasas de variación del $211.6 \%$ y del $141.5 \%$ para hombres y mujeres respectivamente.

Asimismo, la principal característica del desempleo en España viene dada por los desorbitados niveles alcanzados por la tasa de paro entre la población más joven. Los datos reflejan que la tasa de paro entre los menores de 25 años ha pasado del 21,3\% en el primer trimestre de 2008 al 55,1\% en el último trimestre de 2012, lo que se traduce en 0,9 millones de jóvenes sin trabajo.

A pesar de que las tasas de paro juveniles han perjudicado a ambos sexos, el desempleo juvenil ha sido superior en los hombres que en las mujeres. Si bien la tasa de paro masculina para jóvenes menores de 25 años ha aumentado en 36,7 puntos pasando del $19,5 \%$ al $56,2 \%$, la femenina lo ha hecho en 30,3 puntos, pasando del 23,6\% al 53,9\%. Dentro de la población menor de 25 años, las mayores tasas de paro se encuentran en los jóvenes de entre 16 y 19 años, alcanzando el $74,0 \%$ en el cuarto trimestre de 2012, lo que supone un incremento de 41,1 puntos porcentuales respecto a los niveles de 2008. Este hecho pone de manifiesto que los jóvenes tienen serios problemas a la hora de encontrar su primer empleo, principalmente por la falta de experiencia profesional. Asimismo, dentro de este tramo se encuentran también jóvenes que han terminado sus estudios universitarios, y a pesar de que cuentan con altos niveles de cualificación, no encuentran un empleo, lo que supone un grave problema social que tiende a generalizarse.

Otro estrato de población que también se ve seriamente perjudicado por los efectos de la crisis está formado por las personas que se tienen 55 años y más. Durante 2008-2012, este colectivo presentó unas tasas de desempleo en torno al $20,0 \%$. Por sexos, los más perjudicados siguen siendo los hombres, presentando unas tasas de paro del 23,4\% en el cuarto trimestre de 2012 frente al 14,18\% de las mujeres. El principal problema que tienen las personas desempleadas de 55 años y más, es que más de dos tercios son parados de larga duración (AGETT, 2012a; 2012b; 2012c y 2012d), lo que hace que la situación sea especialmente delicada, 
pues el tiempo que de búsqueda de un puesto de trabajo es uno de los factores que más dificulta que se encuentre un nuevo empleo.

\section{El mercado de trabajo relacionado con el sistema de autonomía y atención a la dependencia (SAAD)}

Las cifras anteriores justifican que el mercado de trabajo español está sufriendo las graves consecuencias de una gran crisis financiera y económica. Las pérdidas de empleo y las tasas de paro conducen hacia una situación de malestar social. Si ya la situación no es halagüeña en términos generales si se desglosa por sectores y ramas de actividad la realidad es bastante preocupante, ya que la crisis ha perjudicado a unas actividades mucho más que a otras, como es el caso del sector de la construcción (García Serrano, 2012). Indudablemente este sector y las actividades relacionadas con él han sufrido en mayor medida este efecto.

En el lado completamente opuesto, se encuentran ciertas ramas de actividad, como es el caso de las actividades relacionadas con la atención y promoción de las personas en situación de dependencia ${ }^{2}$ que han sido capaces de generar empleo (véase Camacho Ballesta, et al., 2012; Rodríguez Castedo y Jiménez Lara, 2010; 2011; Sosvilla Rivero, 2009; Herce, et al., 2006).Un empleo, que dependerá de las partidas presupuestarias anuales (véase Montserrat, 2003).

De acuerdo con el informe de síntesis publicado por el IMSERSO "El sistema nacional de dependencia: evaluación de sus efectos sobre el empleo" (Herce, et al., 2006), en el que se muestran diferentes métodos de estimación ${ }^{3}$ del empleo generado por los SAAD, refleja que estas actividades son generadoras de empleo en tiempos de crisis, a pesar de que dichas estimaciones difieran de las cifras ofrecidas por el Libro Blanco de la Dependencia.

Aunque este informe realiza una estimación hasta el año 2010, los años 2011 y 2012 también muestran estas tendencias. En este sentido, los servicios de asistencia en establecimientos residenciales durante el año 2008 ocupaban a 235,4 mil personas y las actividades de servicios sociales sin alojamiento, 153,6 mil ocupados según los datos ofrecidos por la EPA (INE, 2013a). En el año 2012, la asistencia en establecimientos residenciales contaba con 231,0 mil ocupados, y las actividades de servicios sociales sin alojamiento con 207,3 mil trabajadores, lo que supone un incremento total de 49,3 mil empleados entre 2008 y 2012. Por tanto, las actividades vinculadas con la atención a las personas en situación de dependencia se

\footnotetext{
${ }^{2}$ Para poder caracterizar el mercado de trabajo relacionado con la atención a las personas en situación de dependencia, se han utilizado las ramas de actividad 87 (Asistencia en establecimientos residenciales) y 88 (Actividades de servicios sociales sin alojamiento) de la Clasificación Nacional de Actividades Económicas 2009 (CNAE09).

${ }^{3}$ Metodologías Input-Output, macroeconométrica y microeconómica.
} 
catalogan como un yacimiento de empleo en tiempos de crisis porque responde a una doble función, por un lado, paliar el desempleo crónico en nuestra sociedad, y por otro lado, para cubrir necesidades sociales, desatendidas o parcialmente atendidas.

Como muestra el gráfico 2, esta creación de empleo está sujeta a la incorporación y/o trasvases de ocupados de otros sectores económicos que han considerado la atención a las personas en situación de dependencia como una vía para salir de su propia crisis laboral. Del mismo modo, dentro de las propias actividades vinculadas a los SAAD también se produce un trasvase de ocupación (véase gráfico 3).

\section{Gráfico 2. Personas ocupadas en actividades vinculadas a los SAAD. 2008-2012}

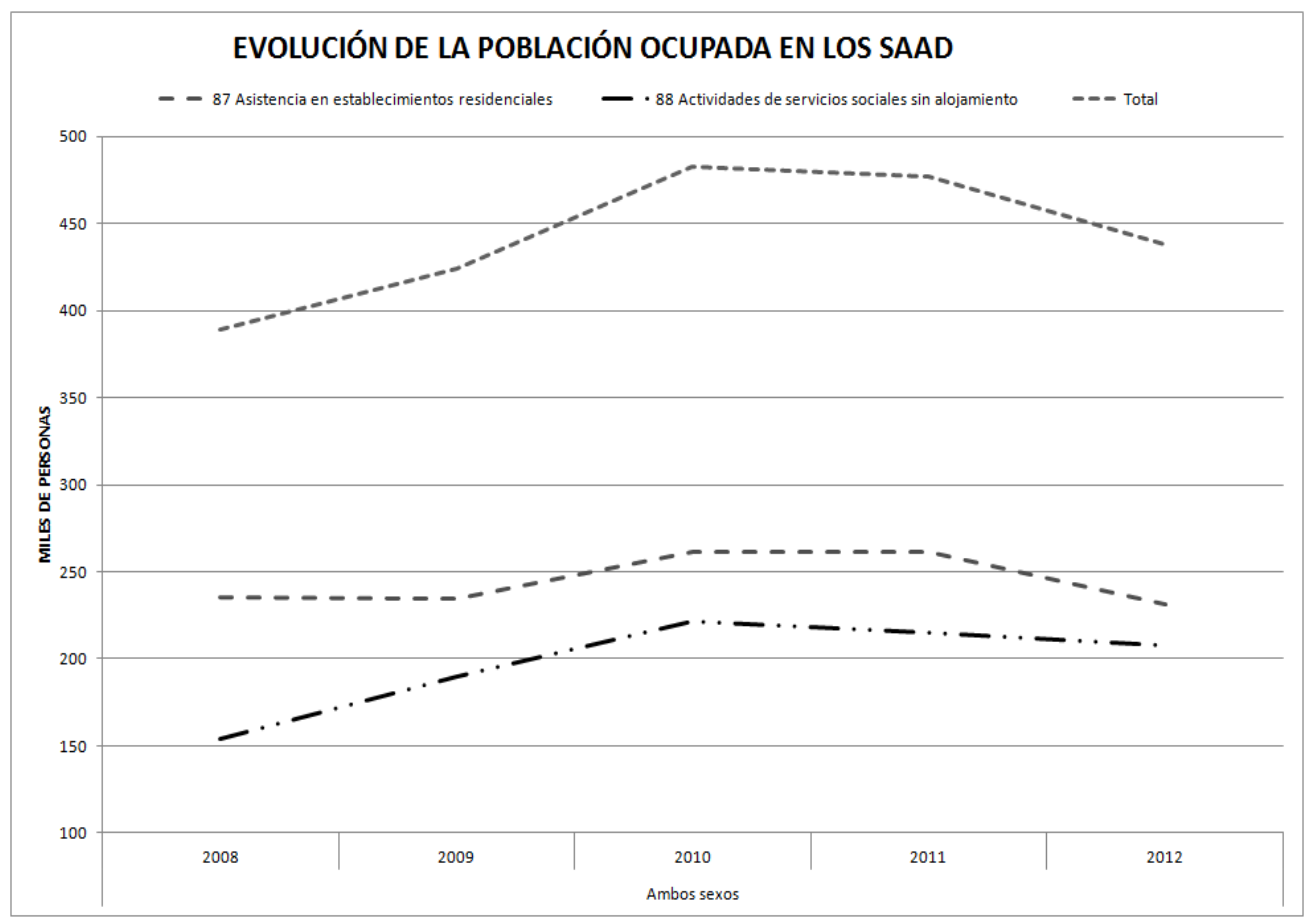

Fuente: Elaboración propia a partir de la EPA (INE, 2013a)

Como muestra el gráfico 3, se observa un aumento en el número de ocupaciones dentro de la asistencia en establecimientos residenciales en detrimento de las actividades de servicios sociales sin alojamiento, sin olvidar la alta participación femenina en este tipo de actividades. De hecho, para 2012, casi nueve de cada diez personas empleadas en actividades de servicios sociales en España eran mujeres, 
repitiéndose este dato desde la implantación de la LAPAD. Esta situación está íntimamente vinculada con el hecho de que

el trabajo asumido tradicionalmente por las mujeres en el ámbito familiar y doméstico les ha permitido desarrollar una serie de aprendizajes, habilidades y competencias que resultan muy útiles para el ejercicio de una actividad profesional remunerada de los cuidados a personas dependientes (Jiménez Lara, 2011:140).

Aunque la implantación de la LAPAD ha dignificado este trabajo, en España (como en otros países mediterráneos),

en los cuidados de larga duración persiste el modelo de bienestar familista, y dentro de la familia este rol corresponde a la mujer. Si bien, las políticas públicas dan por supuesto que las familias deben asumir la provisión de bienestar a sus miembros con la ayuda de una prestación, como mecanismo de protección. De este modo, los sistemas de protección social en países como en España, continúan marcados por añejas connotaciones ideológicas que se sustentas en modelos familiares basados en la división sexual del trabajo (varón sustentador, mujer encargada de las tareas reproductivas y asistenciales), algo que no concuerda con un entorno social cambiante, que en este aspecto penaliza a las mujeres a través del apoyo a la familia, y no se produce un desarrollo adecuado de los servicios sociales oportunos (Rodríguez Rodríguez, 2005:5). 
Gráfico 3. Evolución de los ocupados por sexo y ramas de actividad vinculadas a los SAAD. 2008-2012. Fuente: Elaboración propia a partir de la EPA (INE, 2013a)

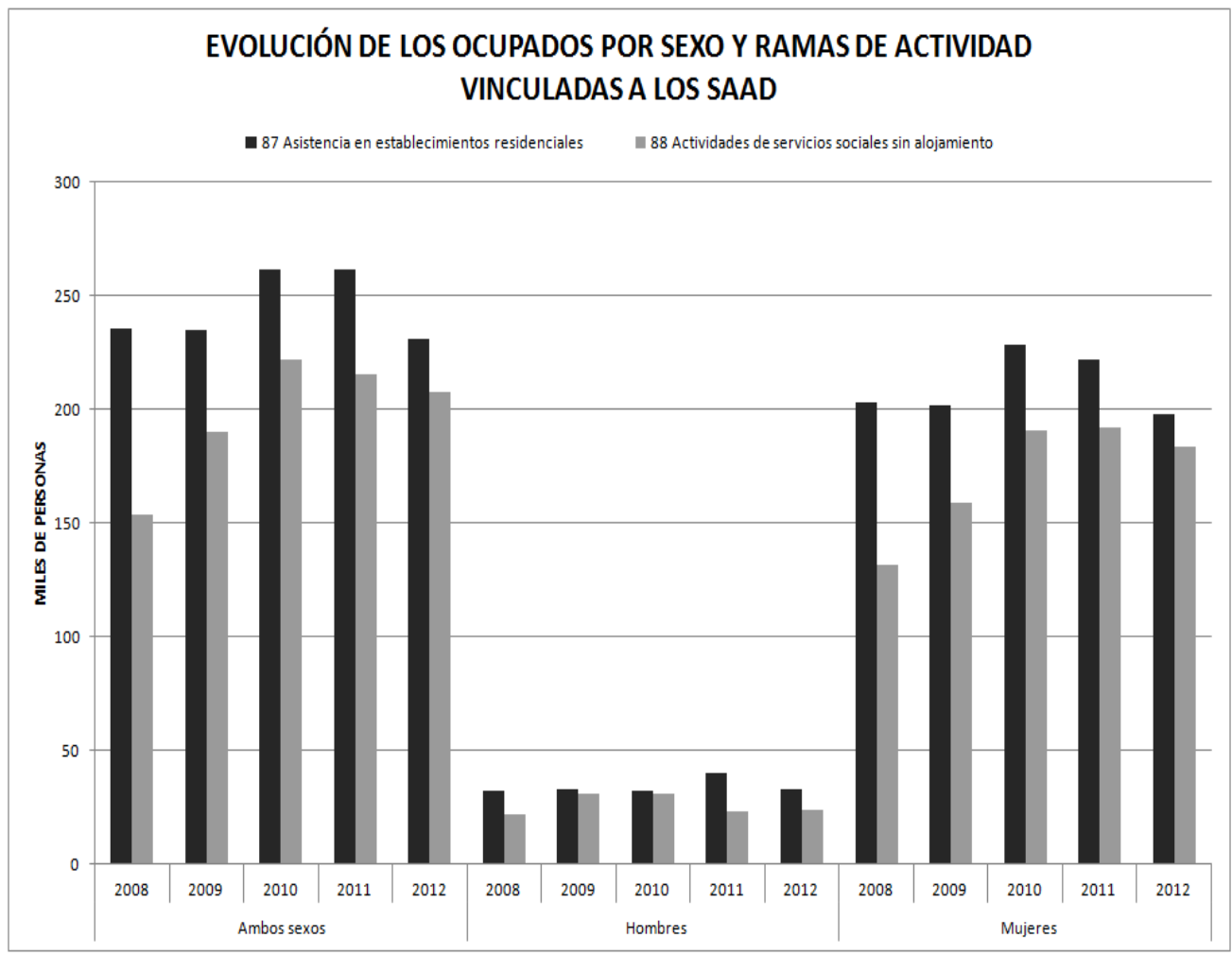

Considerando los datos ofrecidos por la Tesorería General de la Seguridad Social, hay que indicar un cambio de metodología a partir de 2008, por lo que sólo se pueden comparar los datos existentes desde 2009 en adelante. Dichos datos muestran que el total de afiliados en España ha pasado de 13,7 a 12,0 millones entre el 1 de enero de 2009 y el 31 de diciembre de 2012. Las mayores caídas de afiliados se han producido en la construcción donde se ha registrado una pérdida de 372,9 mil afiliados entre 2009-2012.

Si nos centramos en las actividades relacionadas con los SAAD, el número de afiliados ha tenido un comportamiento totalmente diferente, lo que demuestra que son servicios con una demanda creciente y que por tanto se trata de actividades generadoras de empleo. Las actividades de asistencia en establecimientos residenciales han pasado de tener 179,7 mil afiliados en enero de 2009 a 216,9 mil en diciembre de 2012, lo que supone una tasa de variación del 20,7\%. Asimismo, los servicios sociales sin alojamiento han pasado de tener 161,9 mil afiliados en 2009 a 
177,5 mil en el último trimestre de 2012, lo que representa una tasa de crecimiento del 9,6\%.

En el gráfico 4 se muestra la evolución mensual de los afiliados en estas ramas de actividad desde 2009 a 2012. Se observa que el número de personas afiliadas a estas actividades sigue una tendencia o regresión polinómica de segundo grado, mostrando a su vez que el número de afiliados ha crecido considerablemente hasta 2011, año en el que se produce un punto de inflexión, principalmente a partir del segundo cuatrimestre.

En este mismo año se alcanzan máximos de afiliación para ambas actividades (223,2 mil personas afiliadas en julio de 2011 en actividades de asistencia en establecimientos residenciales y 183,2 mil personas en servicios sociales sin alojamiento en diciembre de 2011).

A partir de este año se produce un cambio de tendencia en la que el crecimiento del número de afiliados es más moderado. Asimismo, en dicho gráfico se pone de manifiesto que existe una complementariedad en la evolución de los afiliados de ambas ramas de actividad. En otras palabras, existe una relación indirecta entre el número de afiliados en dichas actividades, si bien un aumento de los afiliados en las actividades de asistencia en establecimientos residenciales supone una reducción de los afiliados en los servicios sociales sin alojamiento y viceversa. A su vez, esta relación se ve corroborada por unos valores de $R^{2}$ próximos a 1 , lo que justifica una alta correlación entre las variables consideradas. 


\section{Gráfico 4. Evolución del número de afiliados en las ramas de actividad vincu- ladas a los SAAD. 2009-2012.}

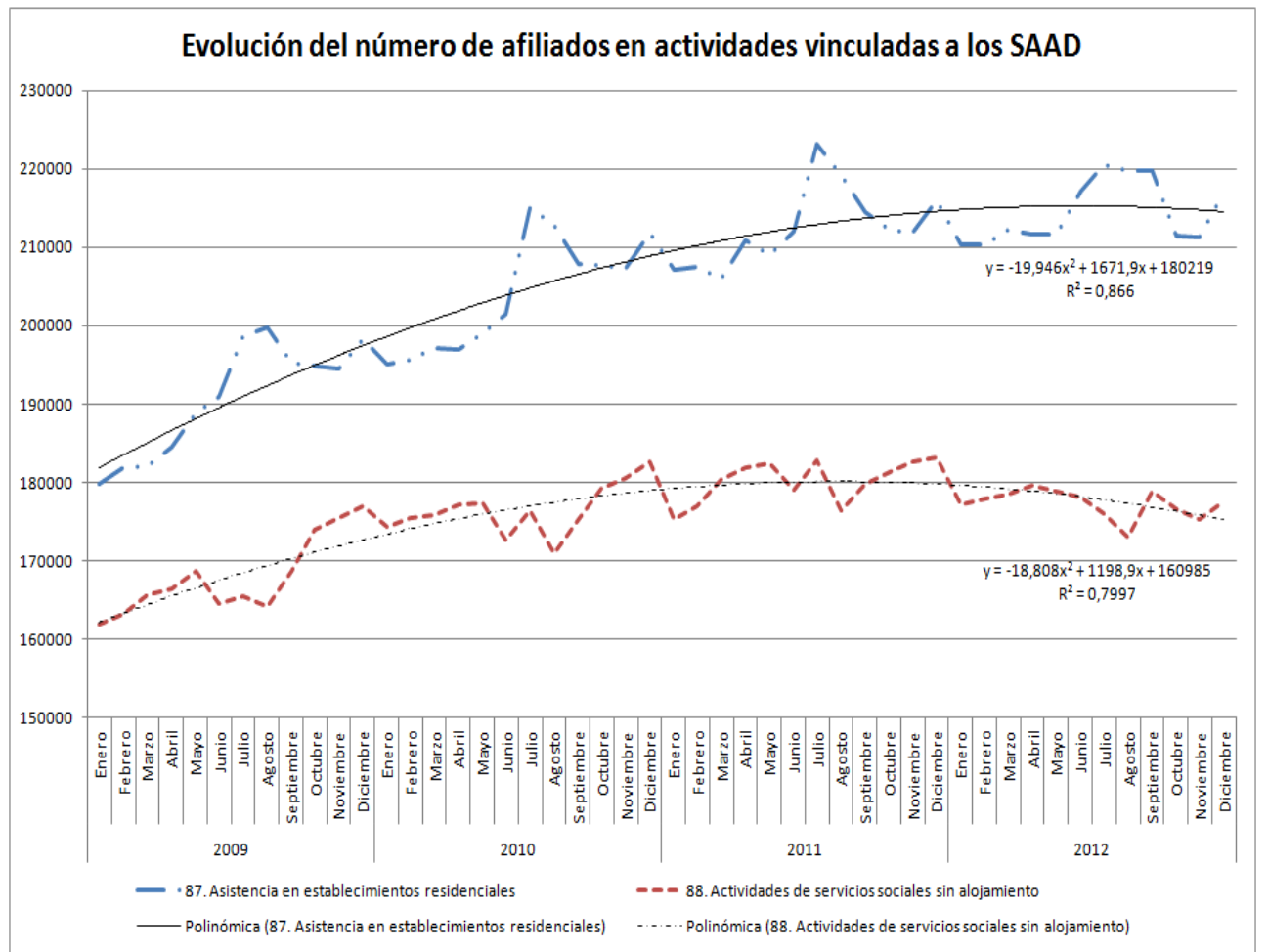

Fuente: Elaboración propia a partir de la Tesorería General de la Seguridad Social (2013)

En España, el crecimiento de las actividades de servicios sociales sin alojamiento está influido por la figura del cuidador no profesional (Camacho Ballesta, et al., 2012). Esta figura está amparada por el Real Decreto 615/2007, de 11 de mayo por el que se regula la Seguridad Social de los cuidadores de las personas en situación de dependencia, según el cual pueden considerarse cuidadores no profesionales de una persona en situación de dependencia, su cónyuge y sus parientes por consanguinidad, afinidad o adopción, hasta el tercer grado de parentesco, salvo cuando la persona en situación de dependencia tenga su domicilio en un entorno caracterizado por insuficiencia de recursos públicos o privados acreditados, despoblación o circunstancias geográficas o de otra naturaleza que impidan o dificulten otras modalidades de atención, en cuyo caso la administración competente podrá excepcionalmente permitir la existencia de cuidados no profesionales por parte de una persona de su entorno que, aun no teniendo el grado de parentesco señalado anteriormente, resida en el municipio de la persona dependiente o en uno vecino, y lo haya hecho durante el periodo previo de un año (Jiménez Lara, 2011). 
Si bien es cierto que en un principio la figura del cuidador no profesional respondería a situaciones puntuales, la práctica ha sido completamente diferente. La creación de esta figura ha supuesto un reconocimiento y una dignificación al trabajo realizado por tantas personas, que han dedicado su vida o parte de ella al cuidado personal desde la esfera privada o familiar, ya que quedan incluidos obligatoriamente en el campo de aplicación del Régimen General de la Seguridad Social y en situación asimilada al alta, mediante la suscripción de un convenio especial.

La mayoría de las personas consideradas como cuidadores no profesionales son mujeres. De hecho, el informe elaborado para el IMSERSO "Dependencia y familia: una perspectiva socio-económica" (De la Torre Prados, et al., 2010), indica que en uno de cada cinco hogares españoles reside una persona con discapacidad, predominando el tipo de hogar de dos miembros donde uno de ellos presenta alguna discapacidad. Se especifica también que el perfil de las personas prestadoras de cuidados a personas en situación de dependencia es una mujer, entre 45 y 64 años que, casi en el $80 \%$ de los casos, reside en el mismo hogar de la persona a la que presta cuidados. Prevalece la figura del cuidador o cuidadora principal (sólo el $23,7 \%$ de los cuidadores son varones) vinculada a la persona dependiente por lazos familiares de consanguinidad.

Según los datos disponibles en la Tesorería General de la Seguridad Social, entre 2008 y 2011 el número de cuidadores no profesionales dados de alta en los últimos días de cada mes, tuvo un incremento en torno al 300,0\% respecto al inicio de la puesta en marcha de esta medida protectora. Sin embargo, el número de personas en alta ha caído drásticamente de los 173.424 en 2011 a los 23.933 en 2012, es decir, un $86,2 \%$. Esta caída se debe a las reformas procedentes del Real Decreto Ley 20/2012 de 13 de julio, de medidas para garantizar la estabilidad presupuestaria y de fomento de la competitividad, en el que se dispone que la cotización será a cargo de la cuidador no profesional, la cual, hasta entonces fue asumida por la Administración General del Estado. Unido todo ello, al descenso en un 15,0\% de la prestación máxima a la que podía acceder.

\section{Conclusiones}

La mejora de la calidad de vida de la sociedad española ha permitido que la población mayor sea más longeva, aumentando con dicha longevidad el número de personas que se encuentran en situación de dependencia, ya que la mayor tasa de prevalencia se encuentra en la población de más de 65 años. Con la finalidad de dar respuesta a una demanda social creciente, como es la atención y mejora de la calidad de vida de las personas en situación de dependencia y la de sus familias, se desarrolló la LAPAD, que no sólo ha cubierto esta necesidad, sino que desde su aplicación en 2007, se han generado 438,3 mil puestos de trabajo(según datos del INE para 2012), 49,3 mil ocupados más respecto a las cifras de 2008, superando 
incluso las expectativas del Libro Blanco de atención a las personas en situación de dependencia.

Desde la implantación de la LAPAD, las mujeres han sido las grandes protagonistas en los servicios vinculados a la atención a la dependencia, y aunque se ha producido un ligero incremento en las colocaciones masculinas, su presencia en estas actividades no resulta significativa. La mayor presencia femenina en este sector, se debe a la persistencia de roles de género asignados culturalmente. De hecho, la "Naturalización" de los roles como cuidadoras dentro ámbito familiar y doméstico culmina con un proceso de socialización orientado al desarrollo de una serie de aprendizajes relacionados con los cuidados, habilidades sociales, modos de relación que se perfilan como una alternativa real de incorporación al mercado de trabajo, a través de una actividad profesional remunerada como son cuidados formales a personas en situación de dependencia (Fernández Villanueva, et al., 2013).

La información procedente de las distintas fuentes estadísticas ratifican que las actividades vinculadas con la atención a las personas en situación de dependencia son un yacimiento de empleo en tiempos de crisis, porque responden a una doble función, por un lado, servir como alternativa para reducir el desempleo crónico en nuestra sociedad, y por otro, cubrir necesidades sociales, desatendidas o parcialmente atendidas hasta entonces.

Finalmente, a pesar de la evidente creación de empleo por parte de este tipo de actividades, no se puede olvidar que dependen en gran parte de las partidas presupuestarias destinadas a tal fin. Unas partidas, que distan de ser estables ante la situación económica por la que atraviesa nuestro país. No obstante, el dinero destinado a mantener las actividades relacionadas con los SAAD no son un mero gasto en bienestar social, sino que se trata de una inversión, ya que promueven el desarrollo económico endógeno de un territorio, en el sentido de que no sólo se crean actividades para atender a las personas en situación de dependencia, sino que sientan las bases para el desarrollo de otras actividades afines y/o complementarias a ellas; estas actividades suponen una fuerza de trabajo no deslocalizable; y sobre todo, procura una cohesión social además de sentar las bases sobre la solidaridad intergeneracional, en la que el envejecimiento progresivo de la población se torna en realidad.

\section{Bibliografía}

Abellán García, A.; Esparza Catalán, C.; Pérez Díaz, J. (2011). Evolución y estructura de la población en situación de dependencia, Cuadernos de Relaciones Laborales, Vol. 29 Núm.1, 2011. doi: 10.5209/rev_CRLA.2011.v29.n1.2

AGETT (Asociación de Grandes Empresas de Trabajo Temporal). (2012a). Informe regional AGETT del mercado laboral $4^{\circ}$ trimestre 2012. Madrid: AGETT. 
AGETT (Asociación de Grandes Empresas de Trabajo Temporal). (2012b). Informe regional AGEET del mercado laboral $3^{\circ}$ trimestre 2012. Madrid: AGETT.

AGETT (Asociación de Grandes Empresas de Trabajo Temporal). (2012c). Informe regional AGETT del mercado laboral $2^{\circ}$ trimestre 2012. Madrid: AGETT.

AGETT (Asociación de Grandes Empresas de Trabajo Temporal). (2012d). Informe regional AGETT del mercado laboral $1^{\circ}$ trimestre 2012. Madrid: AGETT.

Andersen, G. E. (1990). The three worlds of welfare capitalism. Oxford: Polity Press.

A.o.E. (Assembly of European Regions). (2005). The European social model must be grounded in diversity. Consultada en Noviembre 2012 desde http://www.aer.eu/news/2005/2005101301.htm

Beck-Gernsheim, E. (2011). La reinvención de la familia. En busca de nuevas formas de convivencia. Barcelona: Paidós.

Bambra, C. (2007). Going beyond the three worlds of welfare capitalism: Regime theory and public health research, Journal of Epidemiology and Community Health, Vol. 61 Núm. 12, 2007. http://dx.doi.org/10.1136/jech.2007.064295.

Camacho Ballesta, J.; Minguela Recover, M.; Morata García de la Puerta, B.; Hernández Peinado, M.; Ruiz Peñalver, S.; Rodríguez Molina, M. y Sancho Frías, I. (2012). Yacimientos de empleo y políticas sociales: los servicios relacionados con la atención a personas en situación de dependencia en Andalucía. Fundación Pública Andaluza Centro de Estudios Andaluces, Consejería de la Presidencia e Igualdad, Junta de Andalucía.

Camacho Ballesta, J.; Rodríguez Molina, M.; Hernández Peinado, M. (2008). El sistema de atención a la dependencia en España: evaluación y comparación con otros países europeos. Cuadernos Geográficos de la Universidad de Granada, Vol. 40, 37-52.

Carrasco, C. (2006). La paradoja del cuidado: necesario pero invisible. Revista de Economía Crítica, Núm. 5, 39-64.

CE (Council of Europe) (1998). Recommendation $N^{\circ} . R$ (98) 9 of the committee of ministers to member states on dependence.

Del Pozo Rubio, R.; Escribano Sotos, F. (2012). Impacto económico del cuidado informal tras la Ley de promoción de la autonomía personal y atención a las personas en situación de dependencia. Revista Española Salud Pública, Núm. 86, 381-392. 
De la Torre Prados, I., Ruiz Cañete, O., Fernández Moreno, M., Ayuso Sánchez, L., García, L. y Rogero García, J. (2010). Dependencia y familia: una perspectiva socio-económica. Madrid: IMSERSO.

Eikemo, T. A.; Bambra, C.; Judge, K.; \& Ringdal, K. (2008). Welfare state regimes and differences in self-perceived health in Europe: A multilevel analysis, Social Science \& Medicine. Vol. 66 Núm. 11, $2008 . \quad$ doi: 10.1016/j.socscimed.2008.01.022.

Esparza Catalán, C. (2010). Métodos de cálculo de la gravedad de la discapacidad. Informes Portal Mayores. Vol.103, 1-13.

Eurostat (2013a). Health status statistics. Consultada en Octubre, 2013, desde http://epp.eurostat.ec.europa.eu/portal/page/portal/statistics/search_databa se http://epp.eurostat.ec.europa.eu/portal/page/portal/statistics/search_database

Eurostat (2013b). Population projections. Consultada en febrero, 2013, desde http://appsso.eurostat.ec.europa.eu/nui/show.do?dataset=proj_10c2150p\&l ang=en

Fernández Villanueva, C.; Artiaga Leiras, A.; Dávila de León, M.C. (2013). Cuidados, género y transformación de identidades, Cuadernos de Relaciones Laborales, Vol.31 Núm.1, $2013 . \quad$ doi: http://dx.doi.org/10.5209/rev_CRLA.2013.v31.n1.41637

Ferrera, M. (1996). The 'southern model' of welfare in social Europe. Journal of European Social Policy. Vol. 6 Núm.1, 17-37.

Gal, J. (2010). Is there an extended family of Mediterranean welfare states? Journal of European Social Policy. Vol. 20 Núm. 4, 2010. doi: $10.1177 / 0958928710374374$

García Gómez, P.; Jiménez Martín, S.; Vilaplana Prieto, C. (2011). Cuidados no profesionales y atención a la dependencia los cuidados informales. Papeles de economía española. Núm. 129, 83-97.

García Serrano, C. (2012). Del pasmo al marasmo: El sector de la construcción y su relación con la crisis del empleo. Estudios de Economía Aplicada. Vol. 30 Núm. 1, 163-182.

Herce, J.; Labeaga, J.M.; Sosvilla Rivero, S.; Ortega, C. (2006). Sistema Nacional de Dependencia. Evaluación de sus efectos sobre el empleo. Madrid: IMSERSO. 
INE (Instituto Nacional de Estadística) (2012). Proyecciones de Población 2012. Notas de prensa.

INE (Instituto Nacional de Estadística) (2013a). Encuesta de población activa (EPA). Consultada en desde http://www.ine.es/jaxi/menu.do?type=pcaxis\&path=/t22/e308_mnu\&file= inebase $\& \mathrm{~L}=0$

INE (Instituto Nacional de Estadística) (2013b). Proyecciones de población. Notas de prensa.

Jiménez Lara, A. (2011). Haciendo de la necesidad una virtud: la atención a las personas en situación de dependencia como vector del crecimiento del empleo. CIRIEC-España, Revista de Economía Pública, Social y Cooperativa. Vol. 71, 129-146.

Kammer, A.; Niehues, J.; \& Peichl, A. ( 2012). Welfare regimes and welfare state outcomes in Europe. Journal of European Social Polic,. Vol. 22 Núm. 5, 2012. doi: 10.1177/0958928712456572 .

Kemper, P. (1992). The use of formal and informal home care by the disabled elderly. Health Services Research. Vol. 27 Núm. 4, 421-451.

Montserrat Codorniú, J. (2003). El coste de la dependencia. Revista multidisciplinar de gerontología, Vol. 13 Núm. 3, 194-200.

Moreno Rodríguez, L. (2001). La vida media española del modelo de bienestar mediterráneo. Papers: Revista de Sociología. Vol. 63, 67-82.

Prieto, C. y Serrano, A. (2013). Los cuidados entre el trabajo y la vida. Cuadernos de Relaciones Laborales. Vol. 31 Núm. 1, 11-16.

Rodríguez Castedo, A; Jiménez Lara, A. (2010). La atención a la dependencia y el empleo Potencial de creación de empleo y otros efectos económicos de la Ley de Promoción de la Autonomía Personal y Atención a las Personas en Situación de Dependencia. Documento de trabajo (laboratorio de alternativas).Núm.159/2010

Rodríguez Castedo, A.; Jiménez Lara, A. (2011). Nuevas necesidades, nuevos derechos, nuevos empleos: dependencia y creación de empleo. Revista de servicios sociales y política social, Vol. 93, 9-44.

Rodríguez Cabrero, G. (2004). Protección social de la dependencia en España. Madrid: Fundación Alternativas.Núm. 44/2004. 
Rodríguez Cabrero, G. (2007a). La protección social de la dependencia en España. Un modelo sui generis de desarrollo de los derechos sociales. Política y Sociedad, Vol. 44 Núm.2, 69-85.

Rodríguez Cabrero, G. (2007b). El marco institucional de la protección social de la dependencia en España. Estudios De Economía Aplicada. Vol. 25 Núm. 2, 341-372.

Rodríguez Rodríguez, P. (2005). El apoyo informal a las personas mayores en España y la protección social a la dependencia. Del familismo a los derechos de ciudadanía. Revista Española de Geriatría y Gerontología, Vol. 20 Núm. 2, 2005. doi: http://dx.doi.org/10.1016/S0211-139X(05)75068-X

SEPE (Servicio de Empleo Público Estatal) (2013). Datos estadísticos de empleo. Consultada en Febrero, 2013, desde http://www.sepe.es/contenido/estadisticas/datos_estadisticos/empleo/datos/estadisticas_nuevas.html

Sosvilla Rivero, S. (2009). Un análisis estratégico del sistema para la autonomía y atención a la dependencia. Presupuesto y Gasto Público. Vol. 55, 7-29.

Tesorería General de la Seguridad Social (2013). Estadísticas de afiliación y altas de trabajadores. Consultada en Marzo, 2013 desde http://www.segsocial.es/Internet_1/Estadistica/Est/AfiliacionAltaTrabajadores/index.htm 